J. B. Ripoll

Nagoya Math. J.

Vol. 114 (1989), 65-75

\title{
HELICOIDAL MINIMAL SURFACES IN HYPERBOLIC SPACE
}

\author{
JAIME B. RIPOLL
}

\section{§1. Introduction}

Denote by $H^{3}$ the 3-dimensional hyperbolic space with sectional curvatures equal to -1 , and let $g$ be a geodesic in $H^{3}$. Let $\left\{\psi_{t}\right\}$ be the translation along $g$ (see $\S 2$ ) and let $\left\{\varphi_{t}\right\}$ be the one-parameter subgroup of isometries of $\mathrm{H}^{3}$ whose orbits are circles centered on $g$. Given any $\alpha \in R$, one can show that $\lambda=\left\{\lambda_{t}\right\}=\left\{\psi_{t} \circ \varphi_{\alpha t}\right\}$ is a one-parameter subgroup of isometries of $H^{3}$ (see $\S 2$ ) which is called a helicoidal group of isometries with angular pitch $\alpha$. Any surface in $H^{3}$ which is $\lambda$-invariant is called a helicoidal surface.

In this work we prove some results concerning minimal helicoidal surfaces in $H^{3}$. The first one reads:

Theorem A. Let $\alpha \in R,|\alpha|<1$. Then, there exists a one-parameter family $\Sigma$ of complete simply-connected minimal helicoidal surfaces in $H^{3}$ with angular pitch $\alpha$ which foliates $H^{3}$. Furthermore, any complete helicoidal minimal surface in $H^{3}$ with angular pitch $|\alpha|<1$ is congruent to an element of $\Sigma$.

We have the following corollary (see also [An]):

Corollary B. Any complete helicoidal minimal surface in $H^{3}$ with angular pitch $|\alpha|<1$ is globally stable.

The family $\Sigma$ of Theorem 1 allow us to give a characterization of minimal helicoidal surfaces in $H^{3}$, as stated below.

Let $S^{2}(\infty)$ be the Möbius plane, that is, the 2-sphere equipped with the usual conformal structure. Given two points $p_{1}, p_{2}$ in $S^{2}(\infty)$ and $\alpha \in[0, \pi / 2]$, a differentiable curve $\gamma: R \rightarrow S^{2}(\infty)$ which makes an angle $\alpha$ with any circle of $S^{2}(\infty)$ containing $p_{1}$ and $p_{2}$ is called a loxodromic curve with end points $p_{1}$ and $p_{2}$ and with path $\alpha$. By a pair $\left(L_{1}, L_{2}\right)$ of

Received October 27, 1987. 
loxodromic curves we mean two distinct loxodromic curves $L_{1}, L_{2}$ with same path and with same end points.

Now recall that $S^{2}(\infty)$ can be identified with the asymptotic boundary $\partial_{\infty} H^{3}$ of the hyperbolic space $H^{3}$, the conformal structure of $S^{2}(\infty)$ being induced by the extended action of $I S O\left(H^{3}\right)$ to $\partial_{\infty} H^{3}=S^{2}(\infty)$. We prove:

Theorem C. Given any pair of loxodromic curves $\left(L_{1}, L_{2}\right)$ in $S^{2}(\infty)$ with path $\alpha \in[0, \pi / 4)$, there exists one and only one complete properly immersed minimal surface $M^{2}$ in $H^{3}$ such that $\partial_{\infty} M^{2}=L_{1} \cup L_{2}\left(M^{2}\right.$ is congruent to an element of the family $\Sigma$ mentioned in Theorem 1).

The question of determining an immersion in hyperbolic space with constant mean curvature by its asymptotic boundary was first taken up by do Carmo and Lawson ([doCL]). In ([doCGT]), this idea was improved and it has been remarked there the strong influence of the asymptotic boundary of a complete constant mean curvature surface in $H^{3}$ on its global behaviour. In ([LR]), the authors use this idea to characterize catenoids in hyperbolic space and in ([GRR]) is also used to characterize hyperbolic and parabolic surfaces with constant mean curvature in $H^{3}$. We observe that these surfaces, together with the helicoidal ones, exhaust the different types of one-parameter subgroup invariant minimal surfaces in $H^{3}$ (see classification in [R]). We finally remark that in proving Theorem 2, no regularity at infinity has to be assumed, contrary to what happens with similar Theorems (see Theorems 3.1 and 3.2 of [LR], Theorems 2 and 3 of [doCGT] and Theorems 3.3 and 5.2 of [GRR]).

I want to thank Professor Manfredo P. do Carmo who suggested to me the questions about helicoidal minimal surfaces in $H^{3}$.

The results of this paper are part of my doctoral Thesis at IMPA ([R]).

\section{§2. Preliminaries}

We will use the Lorentzian model for the hyperbolic space $H^{3}$, that is,

$$
H^{3}=\left\{\left(x_{1}, x_{2}, x_{3}, x_{4}\right) \mid-x_{1}^{2}+x_{2}^{2}+x_{3}^{2}+x_{4}^{2}=-1\right\},
$$

the Riemannian metric of $H^{3}$ being induced by the quadratic form

$$
q(x)=-x_{1}^{2}+x_{2}^{2}+x_{3}^{2}+x_{4}^{2} \quad x=\left(x_{1}, x_{2}, x_{3}, x_{4}\right)
$$

of $R^{4}$. 
Observe that

$$
\lambda_{t}=\left(\begin{array}{cccc}
\cosh t & \sinh t & 0 & 0 \\
\sinh t & \cosh t & 0 & 0 \\
0 & 0 & \cos \alpha t & -\sin \alpha t \\
0 & 0 & \sin \alpha t & \cos \alpha t
\end{array}\right)
$$

is a one-parameter subgroup of isometries of $H^{3}$ since it preserves $q$, and it is the sum of the translation

$$
\psi_{t}=\left(\begin{array}{cccc}
\cosh t & \sinh t & 0 & 0 \\
\sinh t & \cosh t & 0 & 0 \\
0 & 0 & 1 & 0 \\
0 & 0 & 0 & 1
\end{array}\right)
$$

along the geodesic $g$ : $-x_{1}^{2}+x_{2}^{2}=-1$ plus the rotation

$$
\left(\begin{array}{cccc}
1 & 0 & 0 & 0 \\
0 & 1 & 0 & 0 \\
0 & 0 & \cos \alpha t & -\sin \alpha t \\
0 & 0 & \sin \alpha t & \cos \alpha t
\end{array}\right)
$$

around g. By analogy to the Euclidean space, $\lambda=\left\{\lambda_{t}\right\}$ will be called a helicoidal subgroup of isometries with angular pitch $\alpha$.

Let $P^{2}$ be any totally geodesic 2-submanifold of $H^{3}$ orthogonal to $g$. Let $\vec{o}=P^{2} \cap g$ and define $\rho: P^{2} \rightarrow R$ by $\rho(p)=d(\vec{o}, p), d$ : Riemannian distance. Set $r=\sinh \rho$.

From now on, we choose a geodesic $h$ in $P^{2}$ parametrized by arc length and such that $h(0)=\vec{o}$. Given $p \in P^{2}-\{\vec{o}\}$ denote by $\theta(p)$ the oriented angle between $\vec{p}$ and $h$ where $\vec{p}$ is the geodesic segment from $\vec{o}$ to $p$. $\quad(r(p), \theta(p))$ will be called the polar coordinates of $p$. Computations show that the metric $d s^{2}$ in $P^{2}$ is given in polar coordinates by

$$
d s^{2}=\frac{d r^{2}}{1+r^{2}}+r^{2} d \theta^{2}
$$

It is easy to verify that any orbit of $\lambda$ intersects $P^{2}$ once and just once. Therefore, any $\lambda$-invariant surface is generated by a curve in $P^{2}$. We have the following proposition:

Proposition 2.1. Let $\gamma$ be a curve in $P^{2}$ such that $d \gamma / d t \neq 0$ for any t. Assume that $\gamma$ generates a minimal $\lambda$-invariant surface with angular 
pitch $\alpha$. Then, the polar coordinates $\theta=\theta(t)$ and $r=r(t)$ of $\gamma$ satisfy the differential equation.

$$
\begin{aligned}
& \left(r^{2}+1\right)\left[\left(1+\alpha^{2}\right) r^{2}+1\right]\left(\dot{\theta} \ddot{r}-\dot{r} \ddot{\theta}-r\left(r^{2}+1\right) \dot{\theta}^{3}-\frac{3 r^{2}+2}{r\left(r^{2}+1\right)} \dot{r}^{2} \dot{\theta}\right) \\
& \quad-\left(1+\alpha^{2}\right) r\left(r^{2}+1\right)^{2} \dot{\theta}\left(\frac{\dot{r}^{2}}{r^{2}+1}+r^{2} \dot{\theta}^{2}\right)+2 \alpha^{2} r \dot{\theta}\left(\dot{r}^{2}+r^{2}\left(r^{2}+1\right)^{2} \dot{\theta}^{2}\right)=0 .
\end{aligned}
$$

If $\|\dot{\gamma}\|=1$, then the oriented geodesic curvature $k$ of $\gamma$ is given by:

$$
k=-\frac{\left(1+\alpha^{2}\right)\left(r^{2}+1\right)^{2}-2 \alpha^{2}\left(\dot{r}^{2}+r^{2}\left(r^{2}+1\right)^{2} \dot{\theta}^{2}\right.}{\left[\left(1+\alpha^{2}\right) r^{2}+1\right]\left(r^{2}+1\right)^{3 / 2}} r^{2} \dot{\theta}
$$

Proof. Given $p \in P^{2}$, define $X(p)=(d / d s)\left[\lambda_{s}(p)\right]_{s=0}$ and observe that $\mathscr{B}=\{X(\gamma(t)), d \gamma / d t\}$ is a basis at $\gamma(t)$ of the tangent plane of the surface $S$ generated by $\gamma$. Formula (2.2) is therefore obtained by computing the trace of the second fundamental form of $S$ along $\gamma$ in the basis $\mathscr{B}$. Formula (2.3) is obtained using (2.2) and the formula of the geodesic curvature of a curve in hyperbolic plane.

\section{§3. Description of the helicoidal minimal surfaces}

In this section we study equations (2.1), (2.2) and (2.3) to obtain a description of the helicoidal minimal surfaces.

We begin by observing that the geodesics through $\vec{o}$ in $P^{2}$ generate minimal surfaces (note that they satisfy $\theta=$ constant). As in Euclidean space these surfaces will be called helicoids.

Remark 3.1. Equations (2.1) and (2.2) show that given $p \in P^{2}$ and $v \in T_{p}\left(P^{2}\right),\|v\|=1$, there exists one and only one curve $r$ in $P^{2}$ parametrized by arc length and generating a helicoidal minimal surface with angular pitch $\alpha$ such that $\gamma(0)=p$ and $\dot{\gamma}(0)=v$.

Any such curve will be called a solution curve.

LEMMA 3.2. Let $\gamma$ be a solution curve in $P^{2}$ such that $\dot{r}\left(t_{o}\right)=0$. Let $\tilde{h}$ be a geodesic in $P^{2}$ orthogonal to $\gamma$ at $\gamma\left(t_{0}\right)$. Then $\gamma$ is invariant under the reflexion in $P^{2}$ with respect to $\tilde{h}$.

Proof. Without loss of generality, we may assume $t_{0}=0$. Furthermore, since (2.2) independs on $\theta$, we may also assume that $\theta(0)=0, r=$ $r(t)$ and $\theta=\theta(t)$ being the polar coordinates of $r$. Let $\sigma$ be the reflexion 
on $\tilde{h}$. Then $\tilde{\gamma}=\sigma \circ \gamma$ is given by $\tilde{r}(t)=r(t)$ and $\tilde{\theta}(t)=-\theta(t)+\pi$. Set $\bar{\gamma}(t)=\gamma(-t)$. Therefore, it is easy to verify that the polar coordinates of $\tilde{\gamma}$ and $\bar{\gamma}$ satisfy (2.1) and (2.2). Furthermore, one has $\tilde{\gamma}(0)=\bar{\gamma}(0)$ and $\dot{\tilde{\gamma}}(0)=\dot{\bar{\gamma}}(0)$, that is, $\tilde{\gamma}=\bar{\gamma}$, which proves the Lemma.

Definition 3.3. Let $v$ be a vector field of $P^{2}$ along the geodesic $h$ which is unitary and normal to $h$.

Given $u \in R$, denote by $\gamma_{u}$ the solution curve determined by the initial conditions

$$
\begin{aligned}
& \gamma_{u}(0)=h(u) \\
& \dot{\gamma}_{u}(0)=v(u) .
\end{aligned}
$$

Let $\Gamma=\left\{\gamma_{u}\right\}_{u \in R}$ and $\Sigma=\left\{S_{u}\right\}_{u \in R}$ where $S_{u}$ is the helicoidal minimal surface generated by $\gamma_{u}$.

Remark 3.4. It follows from the above definition and from Lemma 3.2, that any curve $\gamma_{u}$ is invariant with respect to the reflexion on $h$. Also, using Remark 3.1, one can prove that $\gamma_{-u}$ coincides with to the reflexion of $\gamma_{u}$ on the geodesic through $\vec{o}$ of $P^{2}$ orthogonal to $h$.

Lemma 3.5. Any solution curve of $P^{2}$, up to a rotation around $\vec{o}$, belongs to $\Gamma$.

Proof. Let $\gamma$ be a solution curve in $P^{2}$ given in polar coordinates by $\theta=\theta(t)$ and $r=r(t)$. We have just to prove that there exists to such that $\dot{r}\left(t_{o}\right)=0$. By contradiction assume the opposite. Without loss of generality, we may assume that $\lim _{t \rightarrow \infty} r(t)=r_{o} \geq 0$, and we must have $\lim _{t \rightarrow \infty} \dot{r}=0=\lim _{t \rightarrow \infty} \ddot{r}$. If $r_{o}>0$, then, from (2.1) $\lim _{t \rightarrow \infty} \dot{\theta}=\left(1 / r_{o}\right)$. Derivating (2.1) and taking the limit for $t \rightarrow \infty$ we see that $\lim _{t \rightarrow \infty} \ddot{\theta}=0$. But then, taking the limit for $t \rightarrow \infty$ of (2.2) we obtain

$$
\left(r_{o}^{2}+1\right)\left[\left(1+\alpha^{2}\right) r_{o}^{2}+1\right]\left(-\frac{r_{o}^{2}+1}{r_{o}^{2}}\right)-\left(1+\alpha^{2}\right)\left(r_{o}^{2}+1\right)^{2}+2 \alpha^{2}\left(r_{o}^{2}+1\right)^{2}=0
$$

and, after simplifications,

$$
2 r_{o}^{2}+1=0
$$

contradiction!

If $r_{o}=0$, then from (2.1), $\lim _{t \rightarrow \infty} \dot{\theta}=\infty$ and $\lim _{t \rightarrow \infty} r \dot{\theta}=1$. Taking the limit for $t \rightarrow \infty$ of (2.2), we obtain 


$$
\lim _{t \rightarrow \infty} \frac{r \dot{\theta} \ddot{r}-r \dot{r} \ddot{\theta}-r \dot{\theta}^{2}-2 \dot{r}^{2} \dot{\theta}}{r}=1-\alpha^{2}
$$

and then

$$
\lim _{t \rightarrow \infty}\left(r \dot{r} \ddot{\theta}+r \dot{\theta}^{2}+2 \dot{r}^{2} \dot{\theta}\right)=0 .
$$

Derivating (2.1), taking the limit for $t \rightarrow \infty$, we obtain $\lim _{t \rightarrow \infty}(r \dot{r} \ddot{\theta}+$ $\left.\dot{r}^{2} \dot{\theta}\right)=0$, thus

$$
0=\lim _{t \rightarrow \infty}\left(r \dot{\theta}^{2}+\dot{r}^{2} \dot{\theta}\right)=\lim _{t \rightarrow \infty}\left(r \dot{\theta}+\dot{r}^{2}\right) \dot{\theta}=\lim _{t \rightarrow \infty} \dot{\theta}
$$

contradiction!

Theorem 3.6. Any helicoidal minimal surface with angular pitch $\alpha$ is congruent to an element of $\Sigma$.

Proof. Set $\lambda=\left\{\lambda_{t}\right\}_{t \in R}$, and let $S$ be an helicoidal minimal surface with angular pitch $\alpha$. Up to congruence, we may assume that $S$ is $\lambda$ invariant. Hence, it is generated by a curve $\beta$ in $P^{2}$. From Lemma 3.5, there exists a rotation $\tilde{\theta}$ of $P^{2}$ around $\vec{o}$ such that $\tilde{\theta}(\beta) \in \Gamma$. Let $\theta$ be the extension of $\tilde{\theta}$ to $H^{3}$. Then, it is simple to verify that $\theta$ commutes with $\lambda$. Therefore, one has

$$
\theta(S)=\theta(\lambda(\beta))=\lambda(\tilde{\theta}(\beta)) \in \Sigma .
$$

Let $h^{\perp}$ be the geodesic of $P^{2}$ containing $\vec{o}$ orthogonal to $h$.

Proposition 3.7. Assume $|\alpha|<1$. Then, any curve of $\Gamma$ different from $\gamma_{0}$ is a concave graph over $h^{\perp}$.

Proof. Let $\gamma_{u} \in \Gamma, u \neq 0$, and let $\theta=\theta(t)$ and $r=r(t)$ be the polar coordinates of $\gamma_{u}$. To prove the proposition we show that $\theta=\theta(t)$ is a strictly increasing or strictly decreasing function of $t$ and that the geodesic curvature of $\gamma_{u}$ is always positive.

The first statement is obvious since $\dot{\theta}\left(t_{o}\right)=0$ in some point $t_{o}$, then $\gamma_{u}$ would be the geodesic $\theta \equiv \theta\left(t_{o}\right)$ and $u=0$, contradiction.

Since $\dot{r}(0)=0$, from $(2.3)$, we have

$$
k(0)=\frac{\left(1-\alpha^{2}\right) r(0) \sqrt{r^{2}(0)+1}}{\left(1+\alpha^{2}\right) r^{2}(0)+1}
$$

and, since $|\alpha|<1$ and $r(0)>0$, we see that $k(0)>0$.

By contradiction, assume that $k\left(t_{o}\right)=0$ in some point $t_{o}$. Therefore from (2.3) we obtain, at $t=t_{o}$, 


$$
\left(1+\alpha^{2}\right)\left(r^{2}+1\right)^{2}-2 \alpha^{2}\left(\dot{r}^{2}+r^{2}\left(r^{2}+1\right)^{2} \dot{\theta}^{2}\right)=0
$$

hence $\alpha \neq 0$ and

$$
\frac{1+\alpha^{2}}{2 \alpha^{2}}=\frac{\dot{r}^{2}}{\left(r^{2}+1\right)^{2}}+r^{2} \dot{\theta}^{2}
$$

From (2.1), we finally obtain

$$
\left(\frac{r \dot{r}}{1+r^{2}}\right)^{2}=\frac{\alpha^{2}-1}{2 \alpha^{2}}
$$

contradiction!

Definition 3.8. Given $\gamma_{u} \in \Gamma$, let $\theta=\theta_{u}(t)$ be the angular coordinate of $\gamma_{u}$. We define the angle at infinity of $\gamma_{u}$ by $\theta_{\infty}(u)=\lim _{t \rightarrow \infty} \theta_{u}(t)$.

It follows from Proposition 3.7 that $\theta_{\infty}(u) \in(0, \pi / 2]$ for any $u \in[0, \infty)$.

Lemma 3.9. Let $u_{1}, u_{2} \in R, 0<u_{1}<u_{2}$, and let $\theta=\theta_{1}(t)$ and $\theta=\theta_{2}(t)$ be the angular coordinates of $\gamma_{u_{1}}$ and $\gamma_{u_{2}}$, respectively. Assume $|\alpha|<1$ and $\theta_{\infty}\left(u_{2}\right) \leq \theta_{\infty}\left(u_{1}\right)$. Then $\gamma_{u_{1}} \cap \gamma_{u_{2}}=\varnothing$.

Proof. By contradiction, assume $\gamma_{u_{1}} \cap \gamma_{u_{2}} \neq \varnothing$. Therefore, rotating $\gamma_{u_{2}}$ around $\vec{o}$ while keeping fixed $\gamma_{u_{1}}$, there will exist a moment in which $\gamma_{u_{1}}$ and $\gamma_{u_{2}}$ are tangent. But then, $\gamma_{u_{1}}=\gamma_{u_{2}}, u_{1}=u_{2}$, contradiction!

Theorem A stated in the introduction is a consequence of the following result (together with Definition 3.3).

Theorem 3.10. Assume $|\alpha|<1$. Then the family $\Gamma$ foliates $P^{2}$.

Proof. If follows from Proposition 3.7, Remark 3.4 and Lemma 3.9 that we have just to prove that $\theta_{\infty}\left(u_{1}\right)>\theta_{\infty}\left(u_{2}\right)$ if $0<u_{1}<u_{2}$.

Consider the system of differential equations

$$
\begin{aligned}
& \dot{r}=\frac{\operatorname{tr}\left(r^{2}+1\right)\left[\left(1+\alpha^{2}\right) r^{2}+1\right]}{t^{2}\left(4 r^{2}+1+3\left(1+\alpha^{2}\right) r^{4}\right)+\left(r^{2}+1\right)^{2}\left(2 r^{2}+1\right)}, \\
& \dot{\theta}=\frac{\left(r^{2}+1\right)\left[\left(1+\alpha^{2}\right) r^{2}+1\right]}{t^{2}\left(4 r^{2}+1+3\left(1+\alpha^{2}\right) r^{4}\right)+\left(r^{2}+1\right)^{2}\left(2 r^{2}+1\right)} .
\end{aligned}
$$

Assume that $r=r(t)$ and $\theta=\theta(t)$ satisfy $\left({ }^{*}\right)$. Then they verify (2.2). For observe that $\dot{r} / \dot{\theta}=\operatorname{tr}$ so that $(d / d t)(\dot{r} / \dot{\theta})=r+t \dot{r}$, that is, $\dot{\theta} \ddot{r}-\ddot{\theta} \dot{r}=$ $\dot{\theta}^{2}(r+t \dot{r})$ and replace these data in (2.2).

Given $u \in R^{+}$, let $r=r_{u}(t)$ and $\theta=\theta_{u}(t)$ be the solutions of $\left(^{*}\right)$ satisfying 


$$
\begin{aligned}
& r_{u}(0)=u \\
& \theta_{u}(0)=0 .
\end{aligned}
$$

Let $\alpha_{u}$ be the curve in $P^{2}$ given by $\theta=\theta_{u}(t)$ and $r=r_{u}(t)$. It follows from the unicity of the solution curves with respect to the initial conditions that $\alpha_{u}$ is just a reparametrization of $\gamma_{u}$. Now, given $0<u_{1}<$ $u_{2} \in R$, we have $r_{u_{1}}(t) \neq r_{u_{2}}(t)$ for any $t$. Since $r_{u_{1}}(0)=u_{1}<u_{2}=r_{u_{2}}(0)$, we see that $r_{u_{1}}(t)<r_{u_{2}}(t)$ for any $t$. It follows from the expression of $\dot{\theta}$ in (*) that $\dot{\theta}_{u_{1}}(t)>\dot{\theta}_{u_{2}}(t)$ for any $t$. Therefore,

$$
\theta_{\infty}\left(u_{1}\right)=\int_{0}^{\infty} \dot{\theta}_{u_{1}}(t) d t>\int_{0}^{\infty} \dot{\theta}_{u_{2}}(t) d t=\theta_{\infty}\left(u_{2}\right)
$$

which proves the theorem.

Picture. In what follows we use the half-space model for hyperbolic space, namely

$$
H^{3}=\{(x, y, z) \mid z>0\} .
$$

Let $\lambda=\left\{\lambda_{t}\right\}$ be the helicoidal group of isometries which leaves invariant the geodesic axis $z$. We show below a typical surface $S_{u}$.

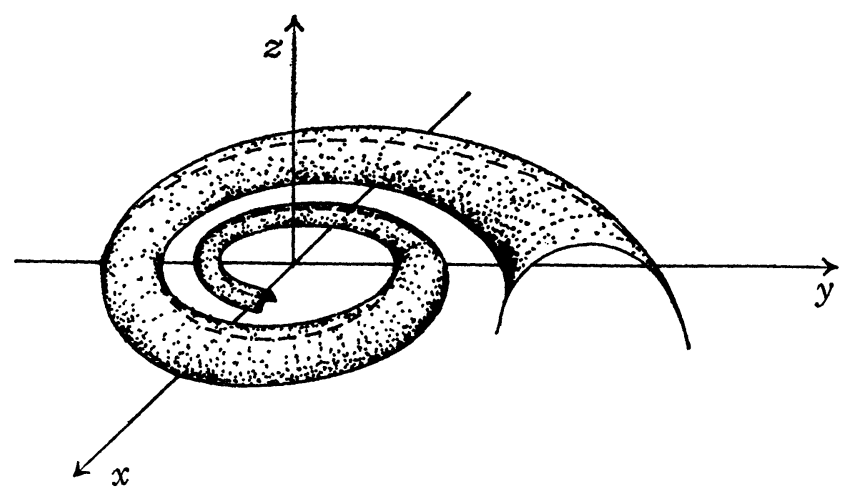

\section{§4. Characterization of the helicoidal minimal surfaces}

In this section we show that an helicoidal minimal surface is determined by its asymptotic boundary (see [doCL]). For, first we prove a result which relates the action of an helicoidal group on the asymptotic boundary of $\mathrm{H}^{3}$ and loxodromic curves.

During this section we will use the half-space model for the hyperbolic space. 
Definition 4.1. Let $p_{1}, p_{2}$ be any two points of $S^{2}(\infty)$ and $\alpha \in[0, \pi / 2]$. A differentiable curve $\gamma: R \rightarrow S^{2}(\infty)$ which makes an angle $\alpha$ with any circle of $S^{2}(\infty)$ containing $p_{1}$ and $p_{2}$ is called a loxodromic curve with ending points $p_{1}$ and $p_{2}$ and path $\alpha$.

Observation 4.2. Let $\lambda=\left\{\lambda_{t}\right\}$ be a helicoidal group of isometries of $H^{3}$ which translation pitch $\alpha$ (that is, $\lambda_{t}=\phi_{\alpha t} \circ \varphi_{t}$, where $\left\{\phi_{t}\right\}$ is a translation along a geodesic $g$ and $\left\{\varphi_{t}\right\}$ the spherical group fixing $g$ ).

Up to conjugation, we may assume that $\lambda$ leaves invariant the geodesic axis $Z$ (in half-space model). Thus, it is not difficult to see that

$$
\lambda_{t}(X, Y, Z)=e^{\alpha t}\left(\left(\begin{array}{rr}
\cos t & -\sin t \\
\sin t & \cos t
\end{array}\right)\left[\begin{array}{l}
X \\
Y
\end{array}\right], Z\right) .
$$

Proposition 4.3. Let $\gamma$ be a differentiable curve in $S^{2}(\infty)$. Then, $\gamma$ is a loxodromic curve if and only if $\gamma$ is the orbit of some point in $S^{2}(\infty)$ under the action of an helicoidal group of isometries of $H^{3}$.

Proof. We can identity $S^{2}(\infty)=\{(X, Y, 0) \mid X, Y \in R\} \cup\{Z=\infty\}$.

Let $\gamma: R \rightarrow S^{2}(\infty)$ be a loxodromic curve with ending points $p_{1}, p_{2}$ and path $\alpha$. Up to a conformal map we may assume that $p_{1}=(0,0,0)$ and $p_{2}=(0,0, \infty)$. Therefore, the circles connecting $p_{1}$ and $p_{2}$ are straight lines through the origin of $R^{2}=\{(X, Y, 0) \mid X, Y \in R\}$.

Observe that the Euclidean structure of $R^{2}$ is compatible with the conformal structure of $S^{2}(\infty)$. Thus, if $\langle$,$\rangle denotes the usual inner-$ product in $R^{2}$, we must have

$$
\frac{\langle\gamma, d r / d t\rangle}{\|\gamma\|\|d r / d t\|} \equiv \cos \alpha=c \quad 0 \leq c \leq 1
$$

If $c=1$ or $c=0$ then $r$ is straight line from $p_{1}$ to $p_{2}$ or a circle centered on $(0,0,0)$, respectively. Therefore, $\gamma$ is the orbit of a translation (helicoidal group with angular pitch 0 ) or $\gamma$ is the orbit of a spherical group (helicoidal group with translation pitch 0 ), respectively.

Assume that $0<c<1$. Setting $\gamma(t)=(X(t), Y(t), 0)$, we obtain

$$
\frac{X \dot{X}+Y \dot{Y}}{\sqrt{X^{2}+Y^{2}} \sqrt{\dot{X}^{2}+\dot{Y}^{2}}}=c .
$$

It is not difficult to show that $\gamma$ can be described by equations of the type: 


$$
\begin{aligned}
X(t) & =r(t) \cos t \\
Y(t) & =r(t) \sin t .
\end{aligned}
$$

Thus, the above differential equation can be easily integrated, providing

$$
\gamma(t)=e^{\beta t}\left(\left[\begin{array}{rr}
\cos t & -\sin t \\
\sin t & \cos t
\end{array}\right]\right)\left(\begin{array}{l}
e^{b} \\
0
\end{array}\right)
$$

where $b$ is a constant and $\beta=c / \sqrt{1-c^{2}}$. This proves the proposition in one direction.

Conversely, given an helicoidal subgroup of isometries $\psi=\left\{\psi_{t}\right\}$, there exists an isometry $g: H^{3} \rightarrow H^{3}$ such that $\psi_{t}=g \lambda_{t} g^{-1}$ (see classification in [R]). The computations above show that the orbits of $\lambda=\left\{\lambda_{t}\right\}$ are loxodromic curves. Thus, given $p \in S^{2}(\infty)$, we have

$$
\psi(p)=\left\{\psi_{t}(p) \mid t \in R\right\}=\left\{g^{-1} \lambda_{t}(g(p)) \mid t \in R\right\}=g^{-1} \lambda(g(p)) .
$$

Since $g^{-1}$ acts conformally in $S^{2}(\infty), \psi(p)$ is also a loxodromic curve.

Definition 4.4. Two loxodromic curves $L_{1}, L_{2} \subset S^{2}(\infty)$ having the same path and the same ending points will be called a pair of loxodromic curves. Notation: $\left(L_{1}, L_{2}\right)$.

It follows from Proposition 4.3 that a loxodromic curve $L$ has path $\alpha$ if and only if $L$ is the orbit of an helicoidal group of angular pitch $\beta=\sin \alpha / \cos \alpha$. In particular $0 \leq \beta<1$ if and only if $0 \leq \alpha<\pi / 4$.

Proof of Theorem C. Up to a conformal map, we may assume that $\left(L_{1}, L_{2}\right)$ has ending points $(0,0,0)$ and $(0,0, \infty)$. Then $\left(L_{1}, L_{2}\right)$ are $\left\{\lambda_{t}\right\}$ invariant. This follows from 4.2 and 4.3. Then, it follows from the hypothesis that $\left\{\lambda_{t}\right\}$ has angular pitch $\alpha$ such that $|\alpha|<1$. Up to a rotation around the $Z$-axis, we may assume that the points $\left\{p_{1}\right\}=\partial_{\infty} P^{2} \cap L_{1}$ and $\left\{p_{2}\right\}=\partial_{\infty} P^{2} \cap L_{2}$ are symmetric with respect to the geodesic $h$ (according to $\S 2$ ).

Now, it follows from Proposition 3.7 and Definition 3.8 that the map $\theta_{\infty}:[0, \infty) \rightarrow(0, \pi / 2]$ is continuous and $1-1$. Then, there exists $u_{0} \in[0, \infty)$ such that $\partial_{\infty} \gamma_{u_{0}}=\left\{p_{1}, p_{2}\right\}$. Hence, $\partial_{\infty} S_{u_{0}}=L_{1} \cup L_{2}$. Clearly, $S_{u_{0}}$ is unique among the minimal complete helicoidal surfaces $\lambda$-invariant.

Let $M \subset H^{3}$ be a complete properly immersed minimal surface such that $\partial_{\infty} M=L_{1} \cup L_{2}$.

Let $p_{+}=h(+\infty)$ and $p_{-}=h(-\infty)$. Since $p_{+} \notin \partial_{\infty} M$, there exists a totally 
geodesic semi-sphere $H^{2}$ in $H^{3}$ centered on $p_{+}$such that $H^{2} \cap M=\varnothing$ and $\partial_{\infty} M \cap \partial_{\infty} H^{2}=\varnothing$. Hence, since $\partial_{\infty} M=L_{1} \cup L_{2}$ is $\left\{\lambda_{t}\right\}$-invariant, we have $\lambda_{t}\left(\partial_{\infty} H^{2}\right) \cap \partial_{\infty} M=\varnothing$ for any $t \in R$. It follows from the Tangency Principle (see [doCL]) that $\lambda_{t}\left(H^{2}\right) \cap M=\varnothing$ for any $t$. Since $\cup_{t \in R} \lambda_{t}\left(H^{2} \cap P\right) \subset$ $\bigcup_{t \in R} \lambda_{t}\left(H^{2}\right)$, it follows that $\left[\bigcup_{t \in R} \lambda_{t}\left(H^{2} \cap P^{2}\right)\right] \cap M=\varnothing$.

$H^{2}$ and $P^{2}$ are totally geodesic submanifolds of $H^{3}$, so that $H^{2} \cap P^{2}$ is a geodesic in $P^{2}$, say $\beta$. Furthermore, since $H^{2}$ in centered on $p_{+}=$ $h(+\infty), \beta$ is orthogonal to $h$. Suppose that $\beta(R) \cap h(R)=\{h(u)\}$. Since the geodesic curvature of $\gamma_{u}$ is always positive, we have $\beta(R) \cap \gamma_{u}(R)=$ $\{h(u)\}$. It follows from the above that $S_{u} \cap M=\left[\bigcup_{t \in R} \lambda_{t}\left(\gamma_{u}(R)\right)\right] \cap M=\varnothing$. Thus, from the Tangency Principle, we obtain $M \cap S_{u}=\varnothing$ for any $u>u_{0}$.

Applying the same arguments considering now the point $p_{-}=$ $h(-\infty)$, we obtain $M \cap S_{u}=\varnothing$ for any $u<u_{0}$. Since $S_{u_{0}}=\lim _{u \rightarrow u_{0}^{+}} S_{u}=$ $\lim _{u \rightarrow u_{0}} S_{u}$, we obtain $M=S_{u_{0}}$.

\section{REFERENCES}

Anderson, M. T., Complete minimal varieties in hyperbolic space, Invent. Math., 69 (1982), 477-494.

do Carmo, M. P. Gomes, J. de M., Thorbergsson, G., The influence of the boundary behaviour on hypersurfaces with constant mean curvature in $H^{n+1}$, Comment. Math. Helv., 61 (1986) , 429-441.

do Carmo, M. P. Lawson, H. B., On Alexandrov-Bernstein theorems in hyperbolic space, Duke Math. J., 50 (1984), 995-1003.

Gomes, J. de M., Ripoll, J. B., Rodríguez L., On surfaces of constant mean curvature in hyperbolic space, preprint (IMPA), 1985.

Levitt, G., Rosenberg, H., Symmetry of constant mean curvature hypersurfaces in hyperbolic space, Duke Math. J., 52, no. 1 (1985).

Ripoll, J. B., Superfícies invariantes de curvatura média constante, Tese de Doutorado, IMPA, 1986.

Universidade Federal do Rio Grande do Sul

Instituto de Matemática

Av. Bento Gonçalves, 9500

91.500-Porto Alegre-RS

Brazil 